\title{
Survival rates of short-span implant-supported cantilever fixed dental prostheses
}

\author{
Abstracted from \\ Aglietta M, Siciliano VI, Zwahlen M, et al. \\ A systematic review of the survival and complication rates of implant supported \\ fixed dental prostheses with cantilever extensions after an observation period of at least 5 years. \\ Clin Oral Implant Res 2009; 20: 441-451 \\ Address for correspondence: Giovanni E Salvi, Department of Periodontology, School of Dental Medicine, \\ University of Bern, Freiburgstrasse 7, CH-3010 Bern, Switzerland. E-mail: giovanni.salvi@zmk.unibe.ch
}

\section{Questions: What are the survival rates of short-span implant-supported cantilever fixed dental prostheses and the incidence of technical and biological complications over 5 years?}

Data sources Publications were sought using Medline, and searches were made by hand of the journals Clinical Oral Implants Research, International Journal of Periodontics and Restorative Dentistry, Journal of Periodontology, Journal of Clinical Periodontology and the International Journal of Oral and Maxillofacial Implants, along with reference lists of identified articles.

Study selection Titles and abstracts were initially screened by two independent reviewers to identify prospective or retrospective longitudinal cohort studies or controlled studies reporting on implantsupported cantilever fixed dental prostheses (ICFDP) with a mean followup period of at least 5 years. A clinical examination had to be performed at the end of the followup. For multiple publications reporting on the same population, only the most recent report was included.

Data extraction and synthesis Data for the meta-analysis were extracted by two independent reviewers. Information regarding survival and complication rates of both implants and ICFDP were extracted. Implant survival was considered if the implant was present at the followup examination; ICFDP survival was considered if the prosthesis was present at the followup visit without any modifications . Peri-implantitis and soft tissue complications were included in the category of biological complications. As for technical complications, all the events affecting the implant and/ or the meso- and/ or the suprastructures' integrity were considered. Among them, the following categories were defined: implant fractures, veneer fractures, framework fractures, abutment or screw fractures, loss of retention and screw loosening.

Results The five studies included in the meta-analysis yielded an estimated 5- and 10-year ICFDP cumulative survival rate of $94.3 \%$ [95\% confidence interval (Cl), 84.1-98\%] and 88.9\% (95\% Cl, 70.8$96.1 \%)$, respectively. Five-year estimates for peri-implantitis were $5.4 \%$ $(95 \% \mathrm{Cl}, 2.0-14.2 \%)$ and $9.4 \%(95 \% \mathrm{Cl}, 3.3-25.4 \%)$ at implant and prosthesis levels, respectively. Veneer fracture (5-year estimate; $10.3 \%$; 95\% Cl, 3.9-26.6\%) and screw loosening (5-year estimate, 8.2\%; 95\% $\mathrm{Cl}, 3.9-17.0 \%)$ represented the most common complications, followed by loss of retention (5-year estimate, $5.7 \% ; 95 \% \mathrm{Cl}, 1.9-16.5 \%$ ) and abutment/ screw fracture (5-year estimate, $2.1 \% ; 95 \% \mathrm{Cl}, 0.9-5.1 \%$ ).
Implant fracture was rare (5-year estimate, 1.3\%; 95\% Cl, 0.2-8.3\%); no framework fracture was reported. Radiographic bone level changes did not yield statistically significant differences either at the prosthesis or at the implant levels when comparing ICFDP with short-span implant-supported end-abutment fixed dental prostheses.

Conclusions ICFDP represent a predictable and reliable treatment for the replacement of posterior missing teeth in partially edentulous patients. The most frequent technical complications included veneer fractures, followed by screw loosening and loss of retention. No detrimental effects on bone levels were observed around implants in the proximity of cantilever extensions. To date, however, evidence is still sparse on the effects of various prosthetic designs (eg, distal or mesial cantilever extension), number of implants supporting ICFDP and occlusal concepts on the incidence of complications in ICFDP.

\section{Commentary}

Although tooth-supported cantilevered fixed partial dentures (FPD) are somewhat controversial in their rates of clinical success, there is consensus that they require more consideration and planning than a conventional FPD. ${ }^{1}$ With the introduction of ICFPD for the completely edentulous arch (ie, Branemark approach), the cantilever has gained acceptance in implant dentistry. ${ }^{2}$ The renewed interest in short-span ICFPD resulting from this acceptance of cantilever design in the completely edentulous arch has led to questions about longer-term survival rates with ICFPD in the partially edentulous patient.

This review sought to analyse survival and complication rates of ICFDP and, in doing so, looked at implant and prosthesis survival rates, defining prosthesis survival as the prosthesis remaining in situ without modifications. Complications were considered biological or technical in nature.

The author's study selection process required that a clinical examination be performed at the end of a followup period of at least 5 years: most of the excluded publications had mean observation periods of $<5$ years or did not provide specific data on ICFDP. Of the five selected studies in this systematic review, only two were specifically designed to test ICFDP. Drawing definitive conclusions about ICFDP longevity from such a small sample size would be inappropriate, but the outcomes do suggest that the short-span ICFDP represents a predictable treatment option when planned correctly.

The most frequently cited technical complications for ICFDP were veneer fracture, screw loosening and loss of retention. These findings are corroborated by more recent studies, but it must be emphasised 
that being mindful of the cantilever length, its functional load and its occlusion will have an impact upon the prosthesis success rate. Although these observations hold true for both tooth and ICFDP, it has been shown that the mere presence of a cantilever extension does not increase the mechanical/ technical risks for implants supporting short-span cantilever FPD. ${ }^{3}$

With crestal bone loss as a significant indicator of implant health, ${ }^{2}$ it was encouraging to see that when ICFDP were compared with implant-supported FPD without cantilevers, there was only a slight difference in the degree of bone loss. Although it was not statistically significant, two out of the five studies that used bone loss as the main indicator of success found that there was more loss around the cantilever extension. Only two out of the five publications reported any biological complications and data were only available for peri-implantitis. No data were reported for periimplant mucositis or soft tissue recession. These disease indicators, certainly important in their own right, should be addressed when looking at implant survival rates. Once again, drawing definitive conclusions from such limited data is problematic.

The authors suggested that in their selected studies there was a considerable variability in outcomes, especially in terms of longterm success rates. This can only lead to the conclusion that, even though there is growing evidence that ICFPD are a viable treatment option, research that is larger in scope will be required before definitive recommendations can be made.

Gary L Stafford

Department of General Dental Sciences, Marquette University School of Dentistry, Milwaukee, Wisconsin, USA

1. Hill EE. Decision-making for treatment planning a cantilevered fixed partial denture. Compendium Cont Ed Dent 2009; 30: 580-585.

2. Misch C. Dental Implant Prosthetics. St Louis: Mosby; 2005.

3. Salvi GE, Bragger U. Mechanical and technical risks in implant therapy. Int J Oral Maxillofacial Implant 2009; 24 (suppl.): 69-84.

\section{Practice points}

- Conventional end-abutment tooth-supported FPD, solely implant-supported FPD or implant-supported single crowns should be the first treatment option. Tooth-implant-supported FPD, tooth-supported FPD with cantilever extensions, and resinbonded fixed reconstructions are to be considered secondary treatment options because of their higher estimated failure rates.

- Using an ICFPD design will reduce treatment time, is more cost-effective, and reduces the risks associated with complex reconstructive surgeries (ie, sinus grafts, anatomical anomalies, ridge augmentation).

Evidence-Based Dentistry (2010) 11, 50-51. doi:10.1038/sj.ebd.6400722 